\title{
Alterstice
}

Revue internationale de la recherche interculturelle

International Journal of Intercultural Research

Revista International de la Investigacion Intercultural

\section{Point de vue des intervenants sur la disproportion des enfants issus de groupes racialisés dans le système de protection de la jeunesse : causes et pistes de solution}

\section{Chantal Lavergne, Sarah Dufour et Dominique Couture}

Volume 4, numéro 1, 2014

URI : https://id.erudit.org/iderudit/1077479ar

DOI : https://doi.org/10.7202/1077479ar

Aller au sommaire du numéro

Éditeur(s)

Alterstice

ISSN

1923-919X (numérique)

Découvrir la revue

Citer cet article

Lavergne, C., Dufour, S. \& Couture, D. (2014). Point de vue des intervenants sur la disproportion des enfants issus de groupes racialisés dans le système de protection de la jeunesse : causes et pistes de solution. Alterstice, 4(1), 17-30. https://doi.org/10.7202/1077479ar

\section{Résumé de l'article}

Alors que l'existence de la disproportion des enfants de certains groupes racialisés dans le système de la protection de la jeunesse est maintenant connue, les raisons précises à l'origine de ce phénomène demeurent encore incertaines. La présente étude fait appel à l'approche qualitative pour comprendre de manière approfondie les facteurs menant à cette disproportion dans le système de protection à Montréal à partir de la perspective de ceux qui interviennent dans les quartiers concernés par ce phénomène, afin d'ouvrir sur des pistes d'action pour remédier à cette situation. L'analyse permet de faire ressortir que la disproportion est envisagée comme un phénomène complexe relié à la fois à l'action des services et des professionnels situés à l'intérieur et à l'extérieur du système de protection de la jeunesse et aux conditions socioéconomiques ainsi qu'au processus d'immigration, d'insertion et d'adaptation à la société d'accueil. Les solutions visant l'amélioration de la situation passent par de véritables changements systémiques au sein des différents organismes agissant auprès des enfants et des familles en difficulté. 


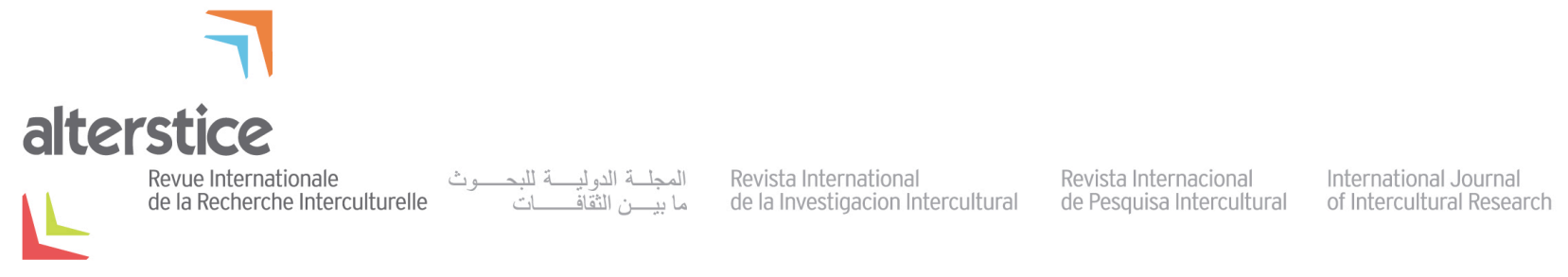

ARTICLE HORS THÈME

\section{Point de vue des intervenants sur la disproportion des enfants issus de groupes racialisés dans le système de protection de la jeunesse : causes et pistes de solution}

Chantal Lavergne $^{1}$, Sarah Dufour ${ }^{2}$ et Dominique Couture ${ }^{1}$

\section{Résumé}

Alors que l'existence de la disproportion des enfants de certains groupes racialisés dans le système de la protection de la jeunesse est maintenant connue, les raisons précises à l'origine de ce phénomène demeurent encore incertaines. La présente étude fait appel à l'approche qualitative pour comprendre de manière approfondie les facteurs menant à cette disproportion dans le système de protection à Montréal à partir de la perspective de ceux qui interviennent dans les quartiers concernés par ce phénomène, afin d'ouvrir sur des pistes d'action pour remédier à cette situation. L'analyse permet de faire ressortir que la disproportion est envisagée comme un phénomène complexe relié à la fois à l'action des services et des professionnels situés à l'intérieur et à l'extérieur du système de protection de la jeunesse et aux conditions socioéconomiques ainsi qu'au processus d'immigration, d'insertion et d'adaptation à la société d'accueil. Les solutions visant l'amélioration de la situation passent par de véritables changements systémiques au sein des différents organismes agissant auprès des enfants et des familles en difficulté.

\footnotetext{
Rattachement des auteurs

${ }^{1}$ Centre jeunesse de Montréal - Institut universitaire, Montréal (Québec), Canada

${ }^{2}$ Université de Montréal, Montréal (Québec), Canada

Cette recherche a été rendue possible grâce au soutien du Conseil de recherche en sciences humaines du Canada (CRSH).
}

\section{Correspondance}

chantal.lavergne@cjm-iu.qc.ca

\section{Mots clés}

disproportion; discrimination; équité; adéquation des services; système de protection de l'enfance

\section{Pour citer cet article}

Lavergne, C., Dufour, S. et Couture, D. (2014). Point de vue des intervenants sur la disproportion des enfants issus de groupes racialisés dans le système de protection de la jeunesse: causes et pistes de solution. Alterstice, 4(1), 17-30. 


\section{Introduction}

La disproportion des enfants issus des groupes racialisés ${ }^{1}$ dans le système de protection de l'enfance est un phénomène qui retient de plus en plus l'attention des chercheurs et des intervenants du domaine de la protection de l'enfance. La disproportion, qui comprend la sur- et la sous-représentation signifie qu'il y a déséquilibre entre la proportion de certains groupes d'enfants aux différentes étapes du système de protection de la jeunesse et leur poids dans la population générale. Ce déséquilibre est préoccupant car il concerne des enfants appartenant à des groupes de population racialisés davantage susceptibles de faire l'objet de traitements discriminatoires ou d'attitudes racistes sur la base de leur couleur, de leur religion ou de leur appartenance ethnoculturelle ou nationale et d'être soumis à différentes formes de désavantages sur le plan socioéconomique (Commission des droits de la personne et de la jeunesse, 2011). Bien que l'appartenance à un groupe racialisé ne résume pas l'ensemble des réalités vécues par les individus qui en font partie, celle-ci constitue néanmoins un facteur susceptible d'influencer de manière importante leur expérience (Thoburn, Chand et Proctor, 2004). La disproportion dans le système de protection peut avoir plusieurs conséquences négatives pour les enfants et leur famille, dont la plus importante est certainement le risque qu'ils fassent l'objet d'un contrôle social accru non justifié ou au contraire d'une intervention inappropriée qui mènerait à tolérer des comportements parentaux dangereux au nom de la culture (Chand, 2000; Dufour, Hassan et Lavergne, 2012). Bien que l'existence d'une disproportion des enfants de groupes racialisés soit relativement bien documentée (Fluke, Jones-Harden, Jenkins et Ruehrdanz, 2011), les raisons exactes à l'origine de ce phénomène demeurent encore méconnues. De plus, les études sur les facteurs responsables de la disproportion reposent principalement sur des méthodologies quantitatives et les résultats demeurent contradictoires (Dettlaff et Rycraft, 2011). On s'est encore peu intéressé au phénomène à partir du point de vue et de l'expérience de ceux qui interviennent dans les quartiers où cette disproportion est importante (Dettlaff et Rycraft, 2011; Hill, 2011). Notre recherche explore les perceptions des professionnels de la protection de l'enfance concernant la disproportion d'enfants de groupes racialisés et les moyens à mettre en œuvre pour réduire l'ampleur de ce phénomène. La première partie résume les connaissances sur l'ampleur et les causes de la disproportion, la deuxième présente la méthode de recherche et la troisième rend compte du point de vue des intervenants sociaux sur les facteurs susceptibles de contribuer à la disproportion dans le système de protection à Montréal, ainsi que sur les pistes de solution envisagées pour remédier à la situation.

\section{L'ampleur de la disproportion}

Aux États-Unis, l'étude d'incidence nationale (NIS) réalisée auprès de diverses catégories de professionnels susceptibles d'entrer en contact avec les enfants constitue la plus importante source de données disponible sur l'ampleur de la maltraitance (Sedlack, McPherson et Das, 2010). Alors que les trois premiers cycles de cette enquête ne trouvent aucune différence entre les groupes de population, les résultats de la quatrième phase de cette étude (NIS-4) révèlent une incidence de maltraitance près de deux fois plus importante pour les enfants noirs que pour les autres enfants (Sedlack et collab., 2010). Une analyse multivariée de ces données montre que les variables associées à la pauvreté expliquent les différences dans les taux de maltraitance entre les deux groupes de population (Sedlack et collab., 2010). Malgré les limites méthodologiques reliées à la mesure du phénomène (Fluke et collab., 2011; Hill, 2006) et l'existence de résultats contradictoires (Hill, 2006), cette disparité dans l'incidence de la maltraitance pourrait néanmoins constituer une piste d'explication possible de la disproportion de ces enfants à l'entrée des services de protection aux États-Unis.

Si l'on mesure mal l'incidence de la maltraitance, on dispose au contraire d'une importante quantité de données sur l'ampleur de la disproportion des enfants des différents groupes racialisés aux principales étapes de décision au sein du système de protection aux États-Unis. Ces décisions concernent la rétention du signalement, la corroboration des faits, l'évaluation de l'existence de motifs de compromission de la situation et le choix des mesures de protection, dont le placement. Selon des statistiques récentes, les enfants afro-américains et autochtones sont surreprésentés, en particulier à l'étape du placement où leur proportion y est de deux à quatre

\footnotetext{
${ }^{1}$ Selon Labelle (2006), le groupe racialisé « renvoie aux groupes porteurs d'une identité citoyenne et nationale précise, mais cibles du racisme ». Figurent notamment parmi ces groupes les communautés noires, arabes, latino-américaines, asiatiques et les nations autochtones.
} 
fois plus importante que leur poids au sein de la population (Hill, 2006; Padilla et Summers, 2011). Une fois placés, ces enfants passent plus de temps en milieu substitut que les enfants blancs (Hines, Lemon, Wyatt et Merdinger, 2004) et ils sont près de quatre fois moins susceptibles que les autres enfants de retourner dans leur milieu familial ou encore d'être adoptés (Hill, 2006). En ce qui a trait aux services offerts, les familles des enfants noirs seraient également moins susceptibles que les autres de bénéficier d'aide en matière de soutien familial, de santé mentale et de toxicomanie (Fluke et collab., 2011). Cependant, tous les groupes racialisés ne sont pas touchés de la même manière par la disproportion : certains enfants, dont les enfants asiatiques, seraient au contraire sous-représentés, et ce, quelle que soit l'étape de prise de décision (Fluke, Yuan, Hedderson et Curtis, 2003; Magruder et Shaw, 2008).

Peu d'études mesurant l'ampleur réelle de la maltraitance selon les différents groupes de population d'enfants ont été réalisées à ce jour au Québec et au Canada. La représentation dans le système de protection est mieux documentée. Les enfants autochtones constituent le groupe le plus touché par ce phénomène (Blackstock, Trocmé et Bennett, 2004; Breton, Dufour et Lavergne, 2012; Lavergne, Dufour, Trocmé et Larrivée, 2008). La surreprésentation de ces enfants est présente à toutes les étapes de décision, mais c'est à celle du placement qu'elle est la plus élevée (Breton et collab., 2012). À Montréal, les rares études portant sur le sujet ont porté principalement sur les enfants haïtiens. Réalisées auprès de petits échantillons, celles-ci ont néanmoins montré que ces enfants faisaient l'objet d'une surreprésentation (Bellony, 2007; Bernard, 2004). On compte encore très peu d'études portant sur les enfants des autres groupes racialisés. En somme, à Montréal comme ailleurs, l'existence d'une disproportion des groupes racialisés dans le système de protection de l'enfance est donc bien établie, mais les raisons à l'origine de ces disparités sont encore mal comprises.

Dans le but de contribuer au développement des connaissances sur cette disproportion, notre équipe de recherche a réalisé une étude comparant différents groupes de population à partir de données sur le signalement de 3918 enfants montréalais (Lavergne, Dufour, Sarmiento et Descôteaux, 2009). Les résultats ont révélé que les enfants noirs (toutes origines confondues) sont surreprésentés, en particulier au seuil du système de protection, où ils sont deux fois plus susceptibles que les autres enfants de faire l'objet d'un signalement. Les enfants des autres groupes racialisés sont, quant à eux, sous-représentés, et ce, quelle que soit l'étape ou la décision analysées. Cependant, au terme de l'évaluation, les enfants noirs et les autres groupes racialisés requièrent moins de services de protection que les enfants non issus de ces groupes. Concernant les mesures de protection, les enfants blancs font plus souvent l'objet d'un placement que les enfants noirs ou ceux issus des autres groupes racialisés. De tels résultats pourraient être attribuables au fait que le profil des enfants blancs et de leur famille présente une plus grande lourdeur sur le plan psychosocial comparativement à celui des enfants issus des groupes racialisés. En outre, les jeunes noirs sont plus souvent orientés vers les services offerts par la communauté.

\section{Causes de la disproportion}

Du point de vue scientifique, plusieurs théories ont été proposées pour rendre compte de la disproportion. On peut regrouper les causes de disproportion selon trois types d'explication : 1) une disparité dans les besoins des familles issues des groupes racialisés, notamment en ce qui a trait à la pauvreté et à l'isolement social, qui augmenterait ou diminuerait le risque de maltraitance et la probabilité d'une intervention de la part des services de protection; 2) la présence de biais culturels et de pratiques discriminatoires chez les professionnels œuvrant dans le domaine de la santé et des services sociaux et 3) des facteurs systémiques reliés aux processus et aux ressources d'aide au sein du système de protection (Fluke et collab., 2011; Hines et collab., 2004; Lemon, D'Andrade et Austin, 2005). À ces trois explications s'ajoutent le contexte géographique et l'environnement communautaire dans lesquels s'inscrivent les organismes de protection, facteurs qui influencent les variations dans l'ampleur et la nature des disparités observées (Fluke et collab., 2011). Les résultats à cet égard demeurent contradictoires et ne permettent pas de statuer de manière définitive sur les causes exactes de ce phénomène. Les disparités observées entre les groupes s'expliqueraient non par un seul type d'explication mais par une combinaison d'explications.

Notre recherche vise à explorer les facteurs menant à la disproportion de certains groupes d'enfants dans le système de protection de la jeunesse à Montréal ainsi que les pistes de solution potentielles en utilisant le point de vue de ceux qui interviennent auprès d'eux. Nous leur avons présenté une étude quantitative sur la disproportion 
réalisée par notre équipe de recherche (Lavergne et collab., 2009), qui a servi à fois comme activité de diffusion des résultats et comme expérience de recherche où de nouvelles connaissances sur les causes de la disproportion et les solutions ont été générées (Duperré, 2006).

\section{Méthode}

\section{Sélection des participants}

Les participants à notre étude étaient intervenants en protection de la jeunesse et travaillaient à Montréal. Ils ont été recrutés sur une base volontaire à partir de deux types de stratégies. Une première stratégie a consisté à relancer l'ensemble des intervenants ayant pris part au volet qualitatif d'une recherche précédente portant sur la réponse du système de protection aux besoins des enfants issus des minorités visibles. Parmi les 24 candidats pressentis, 7 intervenants ont finalement accepté de participer aux entrevues de groupe. Le faible taux de participation s'explique par un refus de participer (4 cas), par un désistement de dernière minute dû à des obligations professionnelles (par exemple, une convocation à la cour) (6 cas) ainsi que par l'impossibilité de rejoindre les intervenants en congé ou ayant changé d'emploi (7 cas). La seconde stratégie a consisté à faire appel aux chefs de service de chaque bureau territorial afin qu'ils fassent parvenir aux intervenants de leur équipe une invitation à participer à la recherche. Cette stratégie a permis d'obtenir la participation de 15 nouveaux intervenants. Au total, 22 intervenants ont donc participé. La plupart étaient des femmes (19) et 15 étaient nés au Canada. Ils possédaient tous une formation universitaire, de premier cycle (17) ou de deuxième cycle (5). Les répondants travaillaient depuis en moyenne 12 ans en protection de la jeunesse (écart-type : 8) et leur expérience variait entre 2 et 29 ans. Ces participants ont dans l'ensemble qualifié leur degré de familiarité avec la clientèle issue des groupes ethnoculturels de " moyen " (15), deux personnes seulement se disant " peu » familières et les 5 dernières « très » familières.

\section{Collecte des données}

L'entrevue de groupe a été privilégiée pour encourager la discussion et l'échange entre les intervenants (Krueger et Casey, 2000). Au total, cinq entrevues de groupe d'environ 60 minutes chacune ont été effectuées. Chaque groupe était composé de 3 à 6 intervenantes, d'une animatrice et d'une assistante. Une version simplifiée du protocole d'entrevue de groupe a été transmise aux participants avant la rencontre afin qu'ils puissent s'y préparer (Patton, 2002). La rencontre a débuté par une brève présentation des résultats de l'étude quantitative réalisée précédemment sur la disproportion des enfants de groupes racialisés dans les services de protection montréalais. Par la suite, deux thèmes ont été abordés. Le premier a concerné les explications possibles à la surreprésentation des enfants noirs et à la sous-représentation des enfants des autres groupes racialisés dans les services de protection de la jeunesse à Montréal. On a demandé aux participants : "Qu'est-ce qui amène les enfants noirs en si grand nombre dans les services de protection? " Le second thème explorait les moyens envisageables pour enrayer ces phénomènes. L'une des questions posées était: "Comment pourrait-on réduire la surreprésentation des enfants noirs aux diverses étapes de la trajectoire de services en Centre jeunesse? " Une courte série de questions sociodémographiques (auxquelles les participants répondaient individuellement) complétait chaque entrevue.

\section{Analyse des données}

Toutes les entrevues ont été enregistrées puis transcrites intégralement en dé-nominalisant les données. L'analyse du contenu des entrevues s'est inspirée de la procédure systématisée par L'Écuyer (1990) : 1) lecture préliminaire et établissement d'une liste d'énoncés à classer; 2) choix et définition des unités de classification (grille de classification) et 3) processus de classification des énoncés. La première étape a débuté par l'analyse horizontale des réponses à chaque question d'entrevue. Un nombre limité de catégories mutuellement exclusives, univoques et homogènes a été créé à partir du contenu explicite du discours des intervenants, puis les transcriptions ont été codées dans ces catégories de réponse à l'aide du logiciel NVIVO. La deuxième étape a visé à comparer et à opposer les catégories les unes aux autres ainsi qu'à identifier les liens entre les divers éléments. II s'agissait de regrouper les catégories en thèmes et sous-thèmes tout en identifiant les régularités et les liens logiques d'articulation entre les idées se dégageant des données. Cette étape a permis l'extraction des trois thèmes principaux: explications de la surreprésentation des enfants noirs, explications de la sous-représentation des enfants issus des autres groupes de population racialisés et stratégies d'action visant la réduction de la 
disproportion. Un accord inter-juges par consensus a été effectué à trois moments lors des analyses. Cette procédure est considérée par les experts comme efficace pour stimuler la réflexion et clarifier les interprétations (Miles et Huberman, 1994).

\section{Résultats}

L'analyse des perceptions des intervenants a permis d'identifier plusieurs facteurs susceptibles de contribuer à la disproportion. Nous présentons d'abord les facteurs contribuant à la surreprésentation, puis ceux contribuant à la sous-représentation. Sont ensuite abordées les pistes de solution énoncées par les répondants.

\section{Point de vue des participants sur les facteurs reliés à la surreprésentation}

Action des services et des professionnels situés à l'intérieur et à l'extérieur du système de protection de la jeunesse

Manque de sensibilité et biais culturels. Selon les participants, les sévices physiques seraient très souvent le motif de signalement évoqué dans le cas des enfants noirs par les déclarants professionnels comme par les enseignants ou les travailleurs sociaux œuvrant en Centre de santé et de services sociaux (CSSS). Sans nier le fait que cette problématique puisse être présente chez bon nombre de familles en difficulté issues de la diversité culturelle, les participants ont estimé que les déclarants ne sont pas à l'abri des problèmes d'interprétation de la situation. La ligne de démarcation entre la correction physique modérée et des gestes abusifs au sens de la loi est souvent ténue. Des problèmes peuvent se poser lorsque les professionnels essaient d'interpréter les comportements ou les intentions des parents de même que les risques encourus par les enfants sans tenir compte du contexte. Dans le cas des enfants noirs, s'ajoute à cette difficulté l'existence de biais culturels qui amènent parfois les déclarants professionnels à présumer, à tort, que les parents ont davantage recours à ce type de méthodes éducatives que les autres.

Un enfant noir dit : « je me fais frapper », tout de suite on téléphone pour faire un signalement, versus un Blanc qui dit : « je me fais frapper $"$, bien OK, on va essayer de comprendre.

Chez les intervenants en protection de la jeunesse, le manque de sensibilité culturelle peut aussi affecter leur évaluation des comportements parentaux et des risques pour l'enfant et, par voie de conséquence, influencer leur décision sur l'existence de motifs de compromission.

Nous [du Centre jeunesse], dans nos repères, on va avoir tendance à parler d'instabilité quand un parent confie son enfant à à peu près tout le monde de la famille. On va dire que c'est un parent qui n'assume pas ses responsabilités. Alors que, pour les parents de ces cultures-là, on sait que ça fait partie pour eux de l'éducation d'un enfant, c'est souvent la famille qui assume l'enfant, et non pas uniquement le parent.

Problèmes reliés à la communication interculturelle. Les participants ont considéré que, dans plusieurs situations d'enfants issus des groupes racialisés qui leur sont signalées, il y aurait dû y avoir plus d'efforts investis par certains déclarants professionnels pour communiquer avec les parents sur ce qui posait problème avec leur enfant. Des problèmes liés à la communication interculturelle entravent en effet souvent la capacité des professionnels à établir avec les familles un lien de confiance, pourtant essentiel pour bien évaluer les risques encourus par l'enfant et développer une relation d'aide avec les parents. Les malentendus, voire les chocs culturels, risquent de fausser l'interprétation des situations et d'amener les professionnels à juger, parfois de manière trop hâtive, que la situation doit être signalée à la Direction de la protection de la jeunesse (DPJ). Le recours au signalement serait alors considéré comme un moyen efficace pour dénouer ce qui peut apparaître aux déclarants comme une impasse.

Ils vont tout de suite peser sur le bouton du signalement, alors que certains problèmes qui pourraient se régler à l'interne... ça fait peur aux gens la DPJ donc ils l'utilisent mais y'a d'autres moyens ... Moi j'me dis que la travailleuse sociale qui est à l'école pourrait lui expliquer le problème que l'enfant vit et cette personne-là, qui est attitrée à l'école, pourrait entrer en communication avec le parent. Ce sont des choses qui pourraient peut-être facilement se régler. 
Manque de temps et d'outils. En ce qui a trait à la DPJ, les participants ont parlé également des efforts et du temps supplémentaires qu'il leur faudrait investir pour obtenir la collaboration des parents issus des autres cultures et pour décider de ne pas retenir le signalement ou encore de fermer le dossier plus rapidement. Certains participants ont jugé ne pas disposer de suffisamment de temps en raison du volume important de situations qu'ils ont à traiter, ce qui les empêche de bien comprendre les difficultés et les besoins des familles issues de l'immigration et des groupes racialisés. Ils se sont plaint également du manque d'outils adéquats pour mieux intervenir dans un tel contexte.

Travailler en contexte multi-ethnique... ça double, ça triple mon intervention. Mais ici, à Montréal, on a quand même les mêmes standards de pratique qu'ailleurs. Alors, je me dis, comme institution, je trouve qu'on n'est pas très outillé... pour nous aider à comprendre ce que c'est de travailler avec les familles pakistanaises, avec les familles sri-lankaises, et la semaine suivante, avec la famille d'Afghanistan... On a beau être capable d'aller chercher l'information sur le contexte culturel, politique, il faut les connaître un peu quand même. Mais on n'a pas beaucoup d'outils pour nous aider ni suffisamment de temps.

\section{Difficultés liées au processus migratoire et à l'insertion dans la société d'accueil}

Pauvreté. Les participants ont mentionné que la pauvreté frappe souvent de manière plus importante les familles noires, ce qui engendre des problèmes sociaux importants susceptibles d'affecter la capacité des parents à prendre soin de leurs enfants et ce qui augmente le risque pour ces derniers d'être signalés à la protection de la jeunesse.

Plus t'es pauvre plus t'as de chances d'avoir un enfant signalé. Les familles noires en général ont un statut socioéconomique plus bas que celui des blancs en général. Ça pourrait expliquer une surreprésentation, pas parce qu'ils sont noirs mais parce qu'ils sont plus pauvres. La pauvreté engendre la négligence, elle engendre plein d'affaires négatives pour les familles...

Processus migratoire. Les participants ont décrit également de nombreux facteurs de stress reliés au processus d'immigration et d'insertion à la société d'accueil, qui fragilisent les familles et rendent parfois nécessaire une intervention de la DPJ.

En plus de la pauvreté, il a d'autres facteurs qui s'ajoutent, qui accentuent et qui amènent une urgence d'intervenir pour nous : c'est toute l'expérience d'immigration. Les parents arrivent ici, puis ils ont tout à penser, je veux dire, leur budget, leur quartier, leur logement, leur travail, etc. C'est très stressant, l'expérience d'immigration.

Isolement social. Les participants ont rappelé aussi que l'immigration entraîne souvent un effet d'isolement important chez certaines familles. Celles-ci se voient privées d'un soutien dont elles pouvaient bénéficier dans leur pays d'origine, notamment pour les soins aux enfants. La perte du réseau de soutien et la difficulté à développer de nouveaux liens sociaux significatifs, combinés à la méconnaissance de la langue et des services d'aide disponibles au sein de la communauté, sont autant de difficultés qui nuisent à l'exercice de la parentalité et qui sont susceptibles d'affecter le bien-être et le développement des enfants.

Une famille qui ne connaît pas beaucoup le monde ici, qui n'a pas de famille, qui n'a pas de réseau, qui ne connaît pas les services, les ressources, etc. peut se retrouver assez facilement démunie et faire en sorte qu'à un moment donné, les enfants vont aller demander de la nourriture au voisin parce qu'ils n'ont rien à manger, puis ça va finir avec un signalement. Mais c'est juste parce qu'ils ne connaissent pas les ressources d'aide et qu'ils n'ont pas les repères.

Manque de connaissances des normes locales. Selon les participants, le manque de connaissance des normes légales et des attentes de la société d'accueil à l'égard des pratiques parentales peut également contribuer à augmenter les risques de signalement aux services de protection et, par voie de conséquence, mener à la surreprésentation de certains groupes d'enfants. Les participants ont donné l'exemple de la correction physique comme méthode éducative ou disciplinaire dont l'usage dans le pays d'origine de certaines communautés serait davantage accepté qu'ici. Selon cette interprétation, les parents de ces familles seraient convaincus que la correction physique est essentielle à l'éducation de leurs enfants. En outre, ceux-ci n'auraient pas reçu l'accompagnement nécessaire pour les aider à intégrer les règles définissant les liens parents-enfants au sein de la société d'accueil et les amener à comprendre pourquoi la punition corporelle est de moins en moins tolérée socialement. 
Je pense qu'il y a un manque d'éducation, un manque d'information, ce qui amène, dans le fond, à poursuivre dans la lignée de ce qu'ils savent, de ce qu'ils connaissent, de corriger physiquement les enfants, c'est leur moyen.

\section{Point de vue des participants sur les facteurs reliés à la sous-représentation}

\section{Identification et réponse aux mauvais traitements}

Plusieurs participants considèrent que la sous-représentation de certains groupes d'enfants dans le système de protection de l'enfance peut s'avérer tout aussi préoccupante que la surreprésentation. Elle peut refléter la situation d'enfants ayant besoin de protection mais n'ayant pas été repérés, du fait d'un écart de perception entre la société d'accueil et les membres des groupes visés, par exemple à propos de ce qui constitue un mauvais traitement ou encore de la manière de réagir aux difficultés familiales.

Une tante issue des minorités visibles va avoir tendance à régler le problème au sein de la famille. Elle va [...] appeler toute la famille [...] pour que le secret ne sorte pas à l'extérieur de la famille. [...] Au lieu d'aller à l'extérieur pour chercher de l'aide, on va essayer de voir avant ce qu'on peut faire. C'est parce qu'ils sont en mesure de compter sur le réseau familial c'est comme ça qu'ils ont grandi, puis c'est comme ça qu'ils voient la chose et qu'ils règlent leurs problèmes.

Selon les répondants, certains membres des groupes racialisés sont aussi moins portés à faire appel à la DPJ pour obtenir de l'aide, soit parce qu'ils se méfient des organismes publics ou des services sociaux, auxquels ils attribuent uniquement un rôle de répression, soit parce qu'ils ne connaissent pas la loi ni leurs devoirs de citoyen à cet égard.

II y a toute la question de l'État, tu sais, la DPJ fait partie de l'État au même titre que la police fait partie de l'État. Et l'État c'est gros, parce que, chez eux, l'État c'est...[menaçant].

\section{Facteurs de protection}

À l'inverse, la sous-représentation pourrait s'expliquer par l'influence de facteurs de protection spécifiques à certaines communautés, facteurs qui diminueraient l'occurrence de mauvais traitements. Comme certains participants l'ont évoqué, il est possible que les valeurs et les systèmes de croyances de certaines communautés, qui valorisent l'harmonie et le respect entre les membres de la famille, puissent contribuer à inhiber les sévices physiques ou l’occurrence de négligence envers les enfants.

Ils vont à l'école ces enfants-là, ils vont voir un médecin, ils vont dans les CLSC [Centres locaux de services communautaires]. Alors comment se fait-il qu'ils ne sont pas signalés? Alors à quelque part il y a quelque chose qui fait qu'ils n'ont pas besoin de la DPJ là... ils ont une façon de faire qui est positive avec les enfants.

\section{Point de vue des participants sur les pistes de solution pour éliminer la disproportion}

Les participants ont mentionné différentes pistes de solutions susceptibles de contribuer à l'élimination de la disproportion. Celles-ci ont été regroupées en deux catégories : celles ciblant l'action des professionnels et des services et celles ciblant les facteurs de risque associés aux mauvais traitements envers les enfants.

\section{Pistes ciblant l'action des professionnels et des services}

Sensibilisation et formation des professionnels. Les participants ont considéré comme important que des actions soient entreprises auprès de leurs partenaires pour diminuer la surreprésentation à l'entrée du système de protection (étape de la rétention du signalement). Par exemple, ils suggèrent d'améliorer la capacité des déclarants professionnels à mieux reconnaître les indices de maltraitance chez les enfants issus des groupes racialisés en les aidant à distinguer la punition corporelle et les sévices physiques, ou encore la négligence et la pauvreté. Ils proposent également que leurs partenaires soient formés à l'intervention interculturelle, de façon à réduire leurs bais.

Quand j'ai commencé [...] quelques intervenants allaient parler de la négligence, pis des autres problématiques avec les enseignants, le personnel scolaire. On devrait le faire encore, ça s'est un peu perdu. 
Adaptation de l'offre de services en Centre jeunesse. Les participants étaient également convaincus de la nécessité d'adapter l'offre de services en Centre jeunesse sur le plan culturel afin de mieux répondre aux besoins de la clientèle. Ils ont constaté que de nombreux efforts ont été faits, mais que beaucoup reste encore à accomplir. Parmi les stratégies identifiées, on retrouve, tout d'abord, l'importance pour les intervenants de développer leur compétence « culturelle » de façon à mieux comprendre « la portée de ce qu'on fait, de voir aussi comment, dans nos actions on respecte ou non le parent. Les conséquences aussi que notre intervention peut avoir sur la famille ».

Un participant a soulevé aussi l'importance d'effectuer l'évaluation des risques de compromission encourus par les enfants en distinguant ce qui relève de la culture, du processus migratoire et des comportements dysfonctionnels des parents, pour éviter de tomber dans le piège du relativisme culturel ou dans celui de l'intransigeance à l'égard de toute forme d'adaptation de l'intervention aux besoins des familles.

Sur le plan organisationnel, certains participants souhaitaient une meilleure reconnaissance du fait qu'intervenir en contexte interculturel exige plus de temps. Cette prise en compte pourrait se traduire, par exemple, par une diminution du nombre de cas à leur charge pour leur permettre d'investir le temps et les efforts nécessaires pour améliorer la communication avec les familles et augmenter ainsi l'efficacité de leur intervention.

L'intervention interculturelle, cela demande plus de temps. Je me dis aussi qu'en tant qu'institution, il faudrait en tenir compte un peu. Travailler avec des interprètes, prendre le temps d'expliquer, de démystifier des choses, ça prend plus de temps... alors, il faudrait en tenir compte dans notre charge de cas. Pour mieux intervenir, pour être plus à l'écoute, prendre plus notre temps, bien, il faudrait qu'on puisse le faire...

Les participants ont mentionné aussi l'importance de faire appel aux ressources et de miser sur des outils adaptés culturellement, qu'ils soient déjà existants ou à développer. Ils ont dit utiliser les séminaires interculturels, les aidants naturels issus des communautés et les interprètes qu'ils considèrent, par ailleurs, comme des ressources pertinentes pour faciliter l'accès des familles à des services adaptés culturellement. Plusieurs ont aussi mentionné la formation interculturelle offerte à tous les intervenants de leur établissement comme étant une stratégie essentielle pour favoriser la sensibilité culturelle et améliorer les pratiques. Ils ont recommandé le développement des services, par exemple en élargissant le réseau d'aidants naturels à d'autres communautés ou encore en créant de nouvelles ressources en matière de médiation culturelle, afin de diminuer le recours à la judiciarisation. Les participants ont également souligné l'importance d'avoir une stratégie de communication à l'interne pour mieux faire connaître à tous les intervenants les ressources culturelles déjà disponibles.

Le Centre jeunesse fait de plus en plus d'efforts. Avec la formation multiculturelle, la politique de l'établissement, etc., la prise en compte de la dimension culturelle est plus importante qu'avant. Le recrutement des intervenants qui font partie des minorités visibles aussi. II est aussi possible de faire appel à des interprètes lorsque je ne connais pas certaines choses sur une culture en particulier.

J'ai assisté à une conférence d'une étudiante qui a fait son stage en médiation multiculturelle. Le problème, c'est que ça s'arrêtait là. C'était un projet de stage, un projet de maîtrise; donc, ça n'allait pas plus loin dans le développement de ce service. C'est une des choses que j'aimerais bien que les centres jeunesse adoptent, la médiation interculturelle.

Pistes d'action ciblant les facteurs de risque associés à la maltraitance envers les enfants

Restaurer le lien social. Certains participants ont estimé qu'il est important de restaurer le lien social en servant d'intermédiaire entre les familles et les ressources de la communauté, tout particulièrement dans le cas des familles issues de l'immigration récente ou des minorités qui se méfient des services publics ou les méconnaissent.

Faire appel aux organismes culturellement compétents. Les participants ont aussi noté qu'il est important de faire appel aux organismes culturellement compétents pour favoriser l'ouverture des familles à l'aide offerte. Ces organismes ont en général une meilleure compréhension de la culture des familles et, en particulier, de la manière dont celle-ci influence les croyances des parents concernant la santé, la parentalité et l'éducation des enfants. La fréquentation de ces organismes peut aussi permettre aux familles isolées socialement de développer un lien de confiance avec des personnes significatives susceptibles de leur venir en aide en cas de besoin. 
Il faut que ça passe par les organismes communautaires, qui sont de la même culture qu'elles, ou qui sont très sensibles à leur expérience d'immigration, à leur culture parce qu'ils arrivent à créer un lien de confiance.

Dans les cours de francisation et d'intégration, les profs là-bas, deviennent souvent LA personne qu'ils connaissent d'ici, qui prend le temps de les écouter, et qui fait que, tu sais, ils ont un impact.

Afin de répondre à l'enjeu de la sous-représentation, des participants ont proposé que les services de protection se rapprochent des organismes et des familles qui les fréquentent dans le but de mieux faire connaître la loi et leur action.

On pourrait, par exemple, aller dans des organismes communautaires, faire des rencontres là-bas, faire des séminaires, des présentations auprès des parents pour leur parler de ce qu'on fait à la DPJ. Profiter, par exemple, des festivités des communautés culturelles qui se passent l'été, des affaires comme ça, faire un kiosque, puis nous, la DPJ, voilà notre travail.

Sensibiliser les parents à l'importance de recourir à des méthodes disciplinaires non violentes. Plusieurs participants ont souligné l'importance de travailler sur les méthodes éducatives des parents issus de l'immigration, et ce, dès leur arrivée au Québec, afin de diminuer les risques éventuels de signalement. Ils ont constaté, par ailleurs, que certains établissements et organismes de la communauté font déjà un travail de prévention positif à cet égard et ils ont manifesté le souhait que de telles initiatives soient plus répandues, notamment dans les écoles.

Certaines écoles, sachant qu'il y a un risque, vont faire de l'éducation auprès des parents. Je regarde l'école de ma fille qui couvre une clientèle multiethnique [...] ils sont constamment en éducation avec les parents, avec les nouveaux arrivants.

\section{Discussion}

Selon les intervenants en protection de la jeunesse rencontrés, la disproportion constitue un phénomène complexe, influencé par de multiples facteurs et qui nécessite le déploiement de plusieurs stratégies d'action situées à différents niveaux d'intervention. Les résultats mettent tout d'abord en évidence l'influence des facteurs individuels sur les processus décisionnels au sein des différents systèmes d'aide. Les participants ont souligné à cet égard l'influence des attitudes et des comportements des intervenants au sein des organismes de première ligne sur la décision de signaler ou non les enfants noirs. La présence de biais culturels à l'égard des parents noirs, combinée aux problèmes de communication interculturelle, fait partie des facteurs mentionnés par les participants pour expliquer la tendance plus forte à faire des signalements à la DPJ dans le cas de ces enfants. Le processus de décision à l'intérieur du système de protection n'est pas non plus à l'abri de l'influence des préjugés ou encore de l'ignorance ou de la méconnaissance des autres cultures. Les participants ont en effet mentionné qu'il peut arriver que l'évaluation des risques encourus par les enfants au sein des familles issues de minorités soit faussée car fondée sur une mauvaise interprétation des comportements parentaux. D’autres études qualitatives réalisées aux États-Unis auprès d'informateurs clés font aussi ressortir comme cause à la surreprésentation l'influence des stéréotypes et des préjugés à l'endroit des familles afro-américaines sur les pratiques de signalement ainsi que sur celles liées au placement de ces enfants (Chinball et collab., 2003; Dettlaff and Rycraft, 2008).

Pour prévenir ce problème, les participants ont suggéré de miser sur la formation à l'intervention interculturelle, dans le but d'aider les déclarants professionnels ainsi que les intervenants en protection de la jeunesse à développer leur compétence culturelle. Plusieurs auteurs font aussi de la formation en compétence culturelle une stratégie de choix pour réduire l'influence des biais sur la prise de décision (Chand, 2000; Child Welfare Information Gateway, 2011; Lemon et collab., 2005). Certains auteurs mettent cependant en garde contre une formation centrée exclusivement sur l'intervention interculturelle. Bien que cette approche mette l'accent sur la lutte contre les préjugés et l'enrichissement mutuel par la valorisation de la diversité, celle-ci ne favoriserait pas véritablement l'égalité et le changement social, qui sont garants d'une plus grande équité en matière de services sociaux (Commission des droits de la personne et des droits de la jeunesse du Québec, 2011; Maiter, Staker et Allagia, 2009). Maiter (2009) propose ainsi d'ajouter à l'approche interculturelle un cadre antiraciste axé sur une compréhension des répercussions du racisme sur la vie quotidienne des groupes racialisés ainsi que sur l'adoption de pratiques favorisant le renforcement de l'autonomie et le développement du pouvoir d'agir des familles. Bien 
que la valeur de l'une ou de l'autre de ces approches soit sujette à débat, les résultats de notre étude montrent clairement la nécessité de s'attaquer aux pratiques discriminatoires des professionnels dans le système d'aide.

Les participants ont également insisté sur l'importance d'aider les déclarants professionnels à mieux reconnaître les indices justifiant qu'une situation impliquant une punition corporelle soit signalée ou non à la DPJ. La punition corporelle est un sujet délicat pour les intervenants du milieu de l'éducation et des services sociaux et ceux-ci ne disposent pas toujours des connaissances ni des outils nécessaires pour distinguer clairement les situations de punition corporelle et les situations de sévices physiques. Une recherche qualitative effectuée auprès d'intervenants sociaux a fait ressortir que la très grande majorité des participants avaient eux-mêmes subi la punition corporelle dans leur enfance et ne connaissaient pas l'état actuel des lois encadrant cette pratique (Bélanger-Sabourin, 2011). Plusieurs ont décrit le sentiment d'impuissance qu'ils ont ressenti lors de l'intervention avec des parents convaincus du bien-fondé de cette pratique. Devant ce qui leur est apparu comme étant un refus de collaborer, les intervenants n'ont pas hésité à recourir à leur pouvoir coercitif et à justifier cette décision sur la base d'un discours légal erroné. De tels résultats montrent qu'au-delà de l'information fournie aux déclarants professionnels sur les lois encadrant cette pratique, doit être offert un meilleur soutien à l'intervention auprès des parents qui utilisent de telles méthodes éducatives envers leurs enfants, si l'on veut parvenir à construire une réelle opposition à la punition corporelle chez les familles et prévenir ainsi les situations de sévices physiques qui peuvent en résulter (Aronson Fontes, 2008).

Certains facteurs organisationnels, tels que la non-reconnaissance par l'institution des efforts supplémentaires exigés des intervenants dans ce type de dossiers ou encore le manque d'outils ou de ressources adaptées, ont aussi été identifiés par les participants comme susceptibles de contribuer à la disproportion en protection de la jeunesse. L'impact de tels obstacles sur la qualité des services a aussi été souligné dans plusieurs écrits scientifiques. Les auteurs recommandent différents ajustements, notamment une diminution de la charge de cas, en plus d'une amélioration de l'offre de ressources et de soutien clinique aux intervenants (Chinball et collab., 2003; Hassan et Rousseau, 2007; Lemon et collab., 2005). Bien qu'aucune étude n'ait permis jusqu'à présent d'évaluer les retombées de telles mesures, celles-ci sont néanmoins de plus en plus considérées par les organismes de protection aux États-Unis et même au Québec comme incontournables pour améliorer l'offre de services aux familles de groupes racialisés et contribuer, au moins indirectement, à réduire l'ampleur de la surreprésentation de ces enfants au sein des établissements (Chinball et collab., 2003; Child Welfare Information Gateway, 2011; Dufour, Lavergne, Hassan, Desmonthènes et Savoie, 2010).

Certains participants ont considéré que la surreprésentation est aussi attribuable à l'effet de la pauvreté et du stress engendré par le processus d'immigration et d'adaptation à la société d'accueil. Sans nier l'importance de ces facteurs, certaines données récentes indiquent qu'ils ne seraient pas la première cause de surreprésentation des enfants noirs au seuil des services de protection à Montréal. Une analyse spatiale du lien entre la défavorisation socioéconomique des communautés dans la région montréalaise et le risque de maltraitance indique que ces facteurs joueraient un rôle nettement moins important dans l'explication du signalement des enfants issus des groupes racialisés que dans celui des enfants non issus de ces groupes (Dufour et Lavergne, 2013). Du côté des États-Unis, les études quantitatives réalisées jusqu'à maintenant ne permettent pas d'établir un lien direct entre la pauvreté et la surreprésentation des enfants noirs dans le système de protection. En fait, plusieurs études montrent au contraire que, après contrôle du facteur de la pauvreté, les différences entre les groupes de population sont toujours présentes (Yampolskaya, Armstrong et Vargo, 2007; Wulczyn et Lery, 2007).

Même si la pauvreté et les problèmes familiaux qui en découlent ne peuvent être considérés comme la principale cause de la surreprésentation, ceux-ci constituent néanmoins des facteurs de risque importants de maltraitance envers les enfants (Drake, Lee et Jonson-Reid, 2009; Freisthler, Bruce et Needell, 2007). En ce sens, il est rassurant de constater que les participants à notre étude étaient sensibles au stress considérable associé à la pauvreté et au processus migratoire sur l'expérience de ces familles et à l'importance d'en tenir compte dans les réponses fournies. Les stratégies suggérées à cet égard consistent, entre autres, à aider les familles à briser leur isolement. Recommander aux familles des associations culturelles, des lieux de culte, etc. est considéré comme un moyen pour leur faire connaître les ressources disponibles et les aider à se reconstruire un réseau de soutien et d'entraide (Dettlaff et Rycraft, 2008; Maiter et collab., 2009). Des interventions préventives sont aussi proposées dans le but 
d'offrir aux parents qui ont recours à la punition corporelle un accompagnement particulier. Une telle approche peut s'avérer moins intrusive et stigmatisante qu'une intervention d'autorité en vertu de la Loi sur la protection de la jeunesse (LPJ).

Enfin, pour les participants, la sous-représentation est positive si elle s'explique par l'influence de facteurs de protection spécifiques à certaines communautés, mais elle est inquiétante si elle résulte du fait que certains enfants en besoin de protection ne reçoivent pas l'aide dont ils ont besoin. À cet égard, une étude britannique réalisée auprès de cinq cents familles asiatiques suggèrent que la crainte des répercussions négatives sur l'honneur de la famille et la peur de l'incompréhension, des jugements et du racisme font partie des raisons pour lesquelles celles-ci hésiteraient à signaler la situation d'un enfant en difficulté aux services de protection de l'enfance (NSPCC, 2007). La peur de répercussions légales à un signalement comme l'expulsion, le blocage du processus d'immigration ou la perte de la garde de l'enfant peut également amener certaines familles à éviter de faire appel aux services sociaux pour aider un enfant en difficulté. La sous-représentation peut donc avoir des implications négatives importantes sur la sécurité et le bien-être des enfants. Dans cette perspective, les participants ont considéré qu'il était important que les Centres jeunesse se rapprochent des organismes qui travaillent avec les familles immigrantes et les minorités culturelles, dans le but de démystifier leur mission et leur rôle en matière de protection des enfants et d'aide aux familles et de développer des liens de confiance avec elles. Un tel rapprochement permettrait aussi de mieux faire connaitre la LPJ et les obligations des citoyens et des professionnels à l'égard du signalement et rassurerait les communautés sur le fait que les intervenants sociaux en protection n'ont aucune obligation de dénonciation aux autorités de l'immigration.

\section{Forces et limites}

Notre recherche a permis de donner la parole à des acteurs qui, bien que travaillant quotidiennement dans le système de protection, sont rarement consultés dans les études sur la disproportion. Les entrevues de groupe ont présenté l'avantage d'explorer simultanément les perceptions de plusieurs personnes et d'enrichir ainsi leurs discours respectifs. Par contre, chaque idée a été moins approfondie qu'elle ne l'aurait été en entrevue individuelle. En outre, il peut être plus difficile d'obtenir ainsi des opinions individuelles discordantes ou marginales. De plus, la perspective des participants sélectionnés ne reflète pas nécessairement celle de l'ensemble de ceux qui œuvrent en protection de la jeunesse. Le fait de faire appel au point de vue d'un seul acteur limite également la compréhension que l'on peut dégager des processus de décision, tout particulièrement de ceux reliés au signalement. Enfin, il aurait été intéressant de documenter le point de vue des familles issues des minorités culturelles afin de mieux connaître leur expérience du système d'aide.

\section{Conclusion}

Cette recherche poursuivait un double but: diffuser les résultats d'une étude quantitative antérieure sur la disproportion dans les services de protection montréalais auprès des intervenants qui y travaillent, d'une part, et recueillir leur point de vue sur les causes et les pistes de solution à cette disproportion, d'autre part. Le fait d'être eux-mêmes impliqués professionnellement dans le système concerné par la disproportion a suscité des interprétations fondées sur leur propre expérience, avec ses avantages (comme le réalisme et les exemples concrets) et ses limites (comme une faible mise en perspective). Leur discours converge avec les études antérieures, ce qui suggère que les pistes explicatives et les solutions à la disproportion sont peut-être maintenant connues et que l'amélioration de la situation passe par de véritables changements systémiques au sein des différents organismes agissant auprès des enfants et des familles en difficulté. 


\section{Références bibliographiques}

Aronson Fontes, L. (2008). Child abuse and culture. New York : The Guilford Press.

Bélanger-Sabourin, C. (2011). La punition corporelle comme pratique éducative parentale : représentations sociales d'intervenants sociaux et implications (mémoire de maîtrise). Université du Québec à Montréal, Canada.

Belony, E. (2007). La prise en charge des enfants de l'immigration haïtienne par la Direction de la protection de la jeunesse : une analyse comparative (mémoire de maîtrise). Université du Québec et INRS-UCS, Montréal, Canada.

Bernard, L. (2004). La surreprésentation des jeunes haïtiens dans le système québécois de protection de la jeunesse. Revue Intervention, 160, 117-124.

Blackstock, C., Trocmé, N. et Bennett, M. (2004). Child maltreatment investigations among aboriginal and nonaboriginal families in Canada. Violence against women, 10(8), 901-916.

Breton, A., Dufour, S. et Lavergne, C. (2012). Les enfants autochtones en protection de la jeunesse au Québec : leur réalité comparée à celle des autres enfants. Criminologie, 45, 157-185.

Chand, A. (2000). The over-representation of black children in the child protection system: possible causes, consequences and solutions. Child and family social work, 5, 67-77.

Child Welfare Information Gateway. (2011). Addressing racial disproportionality in child welfare. Washington, DC : U.S. Department of Health and human services, Children's Bureau.

Chinball, S., Dutch, N., Jones-Harden, B., Brown, A et Gourdine, R. (2003). Children of color in the child welfare system: Perspectives from the child welfare community. Washington, DC : U.S. Department of Health and Human Services, Administration for children and families and the Children's Bureau.

Commission des droits de la personne et des droits de la jeunesse du Québec (2011). Profilage racial et discrimination systémique des jeunes racisés. Rapport de la consultation sur le profilage racial et ses conséquences. Montréal : Commission des droits de la personne et des droits de la jeunesse.

Dettlaff, A., et Rycraft, J. (2008). Deconstructing disporportionality: Views from multiple community stakeholders. Child welfare, 87, 37-58.

Dufour, S. et Lavergne, C. (2013). Les signalements d'enfants issus de la diversité culturelle à la protection de la jeunesse. Communication présentée au $6^{\mathrm{e}}$ Colloque québécois sur la maltraitance envers les enfants et les adolescents, Montréal, Canada.

Dufour, S., Hassan, G. et Lavergne, C. (2012). Mauvais traitements et diversité culturelle : bilan des connaissances et implications pour la pratique. Dans Marie-Hélène Gagné, Sylvie Drapeau et Marie-Christine Saint-Jacques (eds, p. 143-165) Les enfants maltraités : de l'affliction à l'espoir. Pistes de compréhension et d'action. Paris : Éditions Solal.

Dufour, S., Lavergne, C., Hassan, G., Demosthène, F. et Savoie, G. (2010). Diversité culturelle et mauvais traitements envers les enfants : pratiques et savoirs. Communication présentée à la $10^{\mathrm{e}}$ édition des conférences GRAVE/CJM-IU, Montréal, Canada.

Duperré, M. (2006). Innovations sociales dans les organismes communautaires : facteurs intervenant dans le processus de transfert des connaissances. Montréal : Université du Québec à Montréal. (Cahiers du centre sur les innovations sociales)

Drake, B., Lee, S., et Jonson-Reid, M. (2009). Race and child maltreatment reporting: Are blacks overrepresented? Children and youth services review, 31(3), 309-316.

Fluke, J., Jones-Harden, B., Jenkins, M. et Ruehrdanz, A. (2011). Research synthesis on child welfare disproportionality and disparities. Washington, DC : American Humane Association and Alliance for Racial Equity in Child Welfare.

Alterstice-Revue Internationale de la Recherche Interculturelle, vol. 4, $n^{\circ} 1$ 
Fluke, J. Yuan, Y.-Y., Hedderson, J. et Curtis, P. (2003). Disproportionate representation of race and ethnicity in child maltreatment: Investigation and victimization. Children and youth services review, 25(5-6), 359-373.

Freisthler, B., Bruce, E. et Needell, B. (2007). Understanding the geospatial relationship of neighborhood characteristics and rates of maltreatment for black, hispanic and white children. Social work, 52(1), 7-16.

Hassan, G. et Rousseau, C. (2007). La protection des enfants: enjeux de l'intervention en contexte interculturel. Bulletin de la recherche interculturelle, 45, 37-50.

Hill, R. (2006). Synthesis of research on disproportionality in child welfare. An update., Washington, DC : Casey-CSSP Alliances for Racial Equity in the child welfare system.

Hill, R. (2011). Responses to a research synthesis on child welfare disproportionality and disparities. Dans Center for the study of social policy and The Annie E. Casey Foundation. Disparities and disproportionality in child welfare: analysis of the research (p. 103-106). Washington, D.C. : American Humane Association and Alliance for Racial Equity in Child Welfare.

Hines, A., Lemon, K., Wyatt, P. et Merdinger, J. (2004). Factors related to the disproportionate involvement of children of color in the child welfare system: a review and emerging themes. Children and youth services review, 26(6), 507-527.

Krueger, R. et Casey, M. (2000). Focus group : A practical guide for applied research ( $3^{\mathrm{e}}$ éd.). Thousand Oaks, CA : Sage.

Labelle, M. (2006). Un lexique du racisme. Étude sur les définitions relatives au racisme et aux phénomènes connexes. Montréal : Université du Québec à Montréal, Observatoire international sur le racisme et les discriminations, Centre de recherche sur l'immigration, l'ethnicité et la citoyenneté.

Lavergne, C., Dufour, S., Trocmé, N. et Larrivée, M.-C. (2008). Visible minority, Aboriginal and Caucasian children investigated by Canadian protective services. Child welfare, 85, 5-31.

Lavergne, C., Dufour, S., Sarmiento, J. et Descôteaux, M.-È. (2009). La réponse du système de protection de la jeunesse montréalais aux enfants issus des minorités visibles. Intervention, 131, 233-241.

L'Écuyer, R. (1990). Méthodologie de l'analyse développementale de contenu. Québec: Presses de l'Université du Québec.

Lemon, K., D'Andrade, A. et Austin, M. (2005). Undersanding and addressing disproportionality in the front end of the child welfare system. Berkeley : Bay Area Social services Consortium, University of California.

Magruder, J. et Shaw, T. (2008). Children ever in care: An examination of cumulative disproportionality. Child welfare, 87(2), 169-188.

Maiter, S. (2009). Using an anti-racist framework for assessment and intervention in clinical practice with families from diverse ethno-racial backgrounds. Clinical social work journal, 37, 267-276.

Maiter, S., Staker, C. et Allagia, R. (2009). The experiences of minority immigrant families : Receiveing child welfare services : Seeking to understand how to reduce risk and increase protective factors. Families in society, $90(1), 28-36$.

Miles, M. et Huberman, M. (1994). Qualitative Data Analysis : An expanded Sourcebook ( $2^{\mathrm{e}}$ éd.). Thousand Oaks, CA : Sage.

NSPCC (2007). Family' honour' dilemma for British Asians reporting child abuse [en ligne]. Revue de presse du 19 mars, consultée le 4 avril 2012 sur le site www.nspcc.org.uk

Padilla, J. et Summers, A. (2011). Disproportionality rates for children of color in foster care, Technical Assistance Bulletin. Reno, NE : National Council of Juvenile and Family Court Judges Permanency Planning for Children, Department of the National Council of Juvenile and Family Court Judges.

Patton, M. (2002) Qualitative research and evaluative methods. Thousand Oaks, CA : Sage. 
Sedlack, A., McPherson, K. et Das, B. (2010). Supplementary analyses of race differences in child maltreatment rates in the NIS-4. Washington, DC : Office of Planning, Research, and Evaluation (OPRE) and the Children's Bureau Administration for Children and Families (ACF), U.S. Department of Health and Human Services (DHHS).

Wulczyn, F. et Lery, B. (2007). Racial disparity in foster care admissions. Chapin Hall : University of Chicago.

Yampolskaya, S., Armstrong, M. et Vargo, A. (2007). Factors associated with exiting and reentry into out of home care under Community-Based Care in Florida. Children and youth services review, 29(10), 1352-1367. 